DOI: $10.15393 /$ j3.art.2015.2910

UDC 517.57

E. G. Ganenkova, V. V. Starkov

\title{
ON REGULARITY THEOREMS FOR LINEARLY INVARIANT FAMILIES OF HARMONIC FUNCTIONS
}

\begin{abstract}
The classical theorem of growth regularity in the class $S$ of analytic and univalent in the unit disc $\Delta$ functions $f$ describes the growth character of different functionals of $f \in S$ and $z \in \Delta$ as $z$ tends to $\partial \Delta$. Earlier the authors proved the theorems of growth and decrease regularity for harmonic and sensepreserving in $\Delta$ functions which generalized the classical result for the class $S$. In the presented paper we establish new properties of harmonic sense-preserving functions, connected with the regularity theorems. The effects both common for analytic and harmonic case and specific for harmonic functions are displayed.
\end{abstract}

Key words: regularity theorem, linearly invariant family, harmonic function

2010 Mathematical Subject Classification: 30C55

1. Introduction. For a function $u(z)$, continuous in the disk $\Delta=\{z \in \mathbb{C}:|z|<1\}$, we denote

$$
M(r, u)=\max _{|z| \leq r}|u(z)| \text { and } m(r, u)=\min _{|z| \leq r}|u(z)| .
$$

Let $S$ be the class of all univalent analytic functions $f(z)=z+\ldots$ in $\Delta$. The theorem of growth regularity asserts that functions having the maximal growth in the given class, grows smoothly (regularly).

Theorem A. [1], [2], [3, pp. 104, 105], 44, pp. 8-9] Let $f \in S$. Then there exist a $\delta_{0} \in[0,1]$ with

$$
\lim _{r \rightarrow 1-}\left[M(r, f) \frac{(1-r)^{2}}{r}\right]=\lim _{r \rightarrow 1-}\left[M\left(r, f^{\prime}\right) \frac{(1-r)^{3}}{1+r}\right]=\delta^{0},
$$

(C) Petrozavodsk State University, 2015 
$\delta^{0}=1$ iff $f(z)=z\left(1-z e^{-i \theta}\right)^{-2}$. If $\delta^{0} \neq 1$, then the functions under the sign of the limit increase on $r$.

If $\delta^{0} \neq 0$, then there exists $\varphi^{0} \in[0 ; 2 \pi)$ such that

$\lim _{r \rightarrow 1-}\left[\left|f\left(r e^{i \varphi}\right)\right| \frac{(1-r)^{2}}{r}\right]=\lim _{r \rightarrow 1-}\left[\left|f^{\prime}\left(r e^{i \varphi}\right)\right| \frac{(1-r)^{3}}{1+r}\right]=\left\{\begin{array}{l}\delta^{0}, \quad \varphi=\varphi^{0} \\ 0, \quad \varphi \neq \varphi^{0}\end{array}\right.$

Here the functions under the sign of the limit are also increasing on $r \in$ $\in(0,1)$.

In [5, Ch. Pommerenke showed that many properties of functions from the class $S$ can be extended to linearly invariant families (LIFs) of locally univalent analytic functions in $\Delta$ of finite order. In [6] and [7, the theorem of growth regularity was obtained for such LIFs.

In [8, 9], the authors introduced the notion of LIF for complex-valued harmonic functions $f$ in $\Delta$. Every such function can be presented, using analytic functions $h$ and $g$ in $\Delta$ in the following way:

$$
f(z)=h(z)+\overline{g(z)}
$$

where

$$
h(z)=z+\sum_{n=2}^{\infty} a_{n}(f) z^{n} \text { and } g(z)=\sum_{n=1}^{\infty} \overline{a_{-n}(f)} z^{n} .
$$

As in [5], L. E. Shaubroek considered locally univalent functions in $\Delta$. Moreover, these functions are sense-preserving in $\Delta$, i. e. the Jacobian $J_{f}(z)$ satisfies

$$
J_{f}(z)=\left|h^{\prime}(z)\right|^{2}-\left|g^{\prime}(z)\right|^{2}>0 \quad \forall z \in \Delta .
$$

Definition 1. 8, 9] A set $\mathfrak{M}_{H}$ of harmonic sense-preserving functions $f$ in $\Delta$ of form (1) is called the linearly invariant family (LIF) if for all $f \in \mathfrak{M}_{H}$ and for any conformal automorphism $\phi(z)=\frac{z+a}{1+\bar{a} z}, a \in \Delta$, the function $e^{-i \theta} f_{a}\left(z e^{i \theta}\right)$ belongs to $\mathfrak{M}_{H}$, where

$$
f_{a}(z)=\frac{f(\varphi(z))-f(\varphi(0))}{h^{\prime}(\varphi(0)) \varphi^{\prime}(0)} .
$$

It is assumed that the order of a family $\mathfrak{M}_{H}$

is finite.

$$
\operatorname{ord} \mathfrak{M}_{H}=\sup _{f \in \mathfrak{M}_{H}}\left|a_{2}(f)\right|
$$


In the analytic case (when $g(z) \equiv 0$ ), the definitions of LIF and ord $\mathfrak{M}_{H}$ coincide with the definitions of Pommerenke [5].

In [10, for LIFs of harmonic functions, the strong order

$$
\overline{\operatorname{ord}} \mathfrak{M}_{H}=\sup _{f \in \mathfrak{M}_{H}} \frac{\left|a_{2}(f)-a_{-1}(f) \overline{a_{-2}(f)}\right|}{1-\left|a_{-1}(f)\right|^{2}}
$$

was defined. The strong order proved to be convenient for investigation of LIFs, because it is not necessary to assume the affine invariance of a family. Moreover, for an affine LIF $\mathfrak{M}_{H}$ the strong order does not exceed the old order:

$$
\operatorname{ord} \mathfrak{M}_{H}-\frac{1}{2} \leq \overline{\operatorname{ord}} \mathfrak{M}_{H} \leq \text { ord } \mathfrak{M}_{H}
$$

This fact allows to describe properties of affine LIFs more precisely. For a LIF $\mathfrak{M}$ of analytic functions, ord $\mathfrak{M}_{H}=\overline{\text { ord }} \mathfrak{M}_{H}$. Analogously to the analytic case in [10] the universal LIF $\mathcal{U}_{\alpha}^{H}$ was introduced and studied. The family $\mathcal{U}_{\alpha}^{H}$ is defined as the union of all LIFs $\mathfrak{M}_{H}$ such that ord $\mathfrak{M}_{H} \leq$ $\leq \alpha$. Equivalently, $\mathcal{U}_{\alpha}^{H}$ is the set of all harmonic sense-preserving functions $f$ in $\Delta$ of the form (1) such that

$$
\overline{\text { ord }} f \stackrel{\text { def }}{=} \overline{\text { ord }}\left\{e^{-i \theta} f_{a}\left(z e^{i \theta}\right): a \in \Delta, \theta \in \mathbb{R}\right\} \leq \alpha .
$$

It was shown in [10] that $\overline{\operatorname{ord}} \mathcal{U}_{\alpha}^{H} \geq 1$.

In [11] and [12, the following regularity theorems for harmonic functions were proved:

Theorem B. (regularity of growth) Let $f \in \mathcal{U}_{\alpha}^{H}$. Set

$$
\begin{gathered}
\Phi_{1}(r)=\int_{0}^{r} M\left(\rho, J_{f}\right) d \rho, \quad \Psi_{1}(r, \varphi)=\int_{0}^{r} J_{f}\left(\rho e^{i \varphi}\right) d \rho, \text { and } \\
F_{1}(r)=\int_{0}^{r} \frac{(1+\rho)^{2 \alpha-2}}{(1-\rho)^{2 \alpha+2}} d \rho .
\end{gathered}
$$

For each $n \geq 2$ successively denote

$$
\Phi_{n}(r)=\int_{0}^{r} \Phi_{n-1}(\rho) d \rho, \quad \Psi_{n}(r, \varphi)=\int_{0}^{r} \Psi_{n-1}(\rho, \varphi) d \rho, \quad \text { and }
$$




$$
F_{n}(r)=\int_{0}^{r} F_{n-1}(\rho) d \rho .
$$

Then

a) for every $\varphi \in[0 ; 2 \pi)$ and $n \in \mathbb{N}$, the functions

$$
\begin{gathered}
J_{f}\left(r e^{i \varphi}\right) \frac{(1-r)^{2 \alpha+2}}{(1+r)^{2 \alpha-2}}, \quad M\left(r, J_{f}\right) \frac{(1-r)^{2 \alpha+2}}{(1+r)^{2 \alpha-2}}, \\
\frac{\Phi_{n}(r)}{F_{n}(r)}, \quad \frac{\Psi_{n}(r, \varphi)}{F_{n}(r)}, \quad \text { and } \frac{\max _{\varphi} \Psi_{n}(r, \varphi)}{F_{n}(r)}
\end{gathered}
$$

are non-increasing on $r \in(0 ; 1)$;

b) there exist constants $\delta^{0} \in[0 ; 1]$ and $\varphi^{0} \in[0 ; 2 \pi)$ such that for $1 \leq n \leq 2 \alpha+2$,

$$
\begin{gathered}
\delta^{0}=\lim _{r \rightarrow 1-}\left[\frac{M\left(r, J_{f}\right)}{J_{f}(0)} \frac{(1-r)^{2 \alpha+2}}{(1+r)^{2 \alpha-2}}\right]=\lim _{r \rightarrow 1-}\left[\frac{J_{f}\left(r e^{i \varphi^{0}}\right)}{J_{f}(0)} \frac{(1-r)^{2 \alpha+2}}{(1+r)^{2 \alpha-2}}\right]= \\
=\lim _{r \rightarrow 1-}\left[\frac{M\left(r, \frac{\partial}{\partial r} J_{f}\right)}{J_{f}(0) 4(\alpha+1)} \frac{(1-r)^{2 \alpha+3}}{(1+r)^{2 \alpha-3}}\right]= \\
=\varlimsup_{r \rightarrow 1-}\left[\frac{\left|\frac{\partial}{\partial r} J_{f}\left(r e^{i \varphi^{0}}\right)\right|}{J_{f}(0) 4(\alpha+1)} \frac{(1-r)^{2 \alpha+3}}{(1+r)^{2 \alpha-3}}\right]= \\
=\lim _{r \rightarrow 1-}\left[\frac{\int_{0}^{r} M\left(\rho, \frac{\partial}{\partial \rho} J_{f}\right) d \rho}{J_{f}(0)} \frac{(1-r)^{2 \alpha+2}}{(1+r)^{2 \alpha-2}}\right]= \\
=\lim _{r \rightarrow 1-}\left[\frac{\int_{0}^{r}\left|\frac{\partial}{\partial \rho} J_{f}\left(\rho e^{i \varphi^{0}}\right)\right| d \rho}{J_{f}(0)} \frac{(1-r)^{2 \alpha+2}}{(1+r)^{2 \alpha-2}}\right]= \\
=\lim _{r \rightarrow 1-} \frac{\Phi_{n}(r)}{J_{f}(0) F_{n}(r)}=\lim _{r \rightarrow 1-} \frac{\Psi_{n}\left(r, \varphi^{0}\right)}{J_{f}(0) F_{n}(r)}=\lim _{r \rightarrow 1-} \frac{\max _{\varphi} \Psi_{n}(r, \varphi)}{J_{f}(0) F_{n}(r)} ;
\end{gathered}
$$

c) $\delta^{0}=1$ for functions $q_{\theta}(z)=e^{i \theta} k_{\alpha}\left(z e^{-i \theta}\right)+\sigma e^{i \theta} \overline{k_{\alpha}\left(z e^{-i \theta}\right)}$, where $\sigma \in \Delta, \theta \in \mathbb{R}$, and

$$
k_{\alpha}(z)=\frac{1}{2 \alpha}\left[\left(\frac{1+z}{1-z}\right)^{\alpha}-1\right] .
$$


Theorem C. (regularity of decrease) Let $f \in \mathcal{U}_{\alpha}^{H}$. Set

$$
Q_{1}(r)=\int_{r}^{1} m\left(\rho, J_{f}\right) d \rho, \quad E_{1}(r)=\int_{r}^{1} \frac{(1-\rho)^{2 \alpha-2}}{(1+\rho)^{2 \alpha+2}} d \rho .
$$

For each $n \geq 2$ successively denote

$$
Q_{n}(r)=\int_{r}^{1} Q_{n-1}(\rho) d \rho, \text { and } E_{n}(r)=\int_{r}^{1} E_{n-1}(\rho) d \rho .
$$

Then

a) for every $\varphi \in[0 ; 2 \pi)$ and $n \in \mathbb{N}$ the functions

$$
J_{f}\left(r e^{i \varphi}\right) \frac{(1+r)^{2 \alpha+2}}{(1-r)^{2 \alpha-2}}, \quad m\left(r, J_{f}\right) \frac{(1+r)^{2 \alpha+2}}{(1-r)^{2 \alpha-2}}, \quad \text { and } \frac{Q_{n}(r)}{E_{n}(r)}
$$

are non-decreasing on $r \in(0 ; 1)$;

b) there exist constants $\delta_{0} \in[1 ; \infty]$ and $\varphi_{0} \in[0 ; 2 \pi)$ such that

$$
\begin{gathered}
\delta_{0}=\lim _{r \rightarrow 1-}\left[\frac{m\left(r, J_{f}\right)}{J_{f}(0)} \frac{(1+r)^{2 \alpha+2}}{(1-r)^{2 \alpha-2}}\right]=\lim _{r \rightarrow 1-}\left[\frac{J_{f}\left(r e^{i \varphi_{0}}\right)}{J_{f}(0)} \frac{(1+r)^{2 \alpha+2}}{(1-r)^{2 \alpha-2}}\right]= \\
=\lim _{r \rightarrow 1-} \frac{Q_{n}(r)}{J_{f}(0) E_{n}(r)}
\end{gathered}
$$

c) for $\varphi \in[0 ; 2 \pi)$ denote

$$
R_{1}(r, \varphi)=\int_{r}^{1} J_{f}\left(\rho e^{i \varphi}\right) d \rho
$$

and for $n \geq 2$, set

$$
R_{n}(r, \varphi)=\int_{r}^{1} R_{n-1}(\rho, \varphi) d \rho
$$

(under the assumptions of Theorem $C$ the integrals converge). If $\delta_{0}<\infty$ then for $n \geq 1$ the function $\frac{R_{n}\left(r, \varphi_{0}\right)}{E_{n}(r)}$ is non-decreasing on $r \in(0 ; 1)$. Moreover,

$$
\delta_{0}=\lim _{r \rightarrow 1-} \frac{R_{n}\left(r, \varphi_{0}\right)}{J_{f}(0) E_{n}(r)}
$$


d) if $J_{f}(z)$ is bounded in $\Delta$, then for every $n \in \mathbb{N}$ and every $\varphi \in[0 ; 2 \pi)$, the functions

$$
\frac{R_{n}(r, \varphi)}{E_{n}(r)} \text { and } \frac{\min _{\varphi} R_{n}(r, \varphi)}{E_{n}(r)}
$$

are non-decreasing on $r \in(0 ; 1)$ and

$$
\delta_{0}=\lim _{r \rightarrow 1-} \frac{\min _{\varphi} R_{n}(r, \varphi)}{J_{f}(0) E_{n}(r)} ;
$$

e) $\delta_{0}=1$ for functions $q_{\theta}(z)=e^{i \theta} k_{\alpha}\left(z e^{-i \theta}\right)+\sigma e^{i \theta} \overline{k_{\alpha}\left(z e^{-i \theta}\right)}$, where $\sigma \in \Delta, \theta \in \mathbb{R}$, and $k_{\alpha}(z)$ is the function defined by (3).

Definition 2. We say that the constant $\varphi^{0}$ from Theorem $B$ is a direction of maximal growth (d.m.g.) of a function $f(z)$. The constant $\varphi_{0}$ from Theorem $C$ is a direction of maximal decrease (d.m.d.) of $f(z)$.

Definition 3. The numbers $\delta^{0}$ from Theorem $B$ and $\delta_{0}$ from Theorem $C$ are called the Hayman numbers of a function $f(z)$.

In the presented paper we establish new properties of $\mathcal{U}_{\alpha}^{H}$, connected with the regularity theorems.

2. Main results. For fixed $c \in[0 ; 1)$ introduce the class $\mathcal{U}_{\alpha, c}^{H}$, consisting of all functions $f=h+\bar{g} \in \mathcal{U}_{\alpha}^{H}$ such that $\left|g^{\prime}(0)\right| \leq c$. That is, $J_{f}(0) \geq 1-c^{2}>0$ for all $f \in \mathcal{U}_{\alpha, c}^{H}$. The class $\mathcal{U}_{\alpha, c}^{H}$ is not a LIF. Note that the family $\mathcal{U}_{\alpha}^{H}$ is not compact in the topology inducted by locally uniform convergence in $\Delta$, but for $\mathcal{U}_{\alpha, c}^{H}$ the following theorem takes place.

Theorem 1. The family $\mathcal{U}_{\alpha, c}^{H}$ is compact in the topology inducted by locally uniform convergence in $\Delta$.

Proof. Let $f_{n} \in \mathcal{U}_{\alpha, c}^{H}, f_{n}=h_{n}+\bar{g}_{n}, n \in \mathbb{N}, h_{n}$ and $g_{n}$ be analytic functions in $\Delta$. By $A_{\alpha}$ denote the set of all analytic functions $h$ in $\Delta$ such that there exists an analytic function $g$ in $\Delta$ and $f=h+\bar{g} \in \mathcal{U}_{\alpha}^{H}$. In other words, $A_{\alpha}$ is the set of analytic parts of functions $f \in \mathcal{U}_{\alpha}^{H}$. The linearly invariance of $\mathcal{U}_{\alpha}^{H}$ implies that $A_{\alpha}$ is a LIF of analytic functions. But for LIFs of analytic functions $\overline{\operatorname{ord}} A_{\alpha}=\operatorname{ord} A_{\alpha}$. Therefore for all $h \in A_{\alpha}$

$$
\left|h^{\prime}(z)\right| \leq \frac{(1+r)^{\alpha-1}}{(1-r)^{\alpha+1}}, \quad|z|=r
$$


see [5]. Since $J_{f}(z)=\left|h^{\prime}(z)\right|^{2}-\left|g^{\prime}(z)\right|^{2}>0$ for all $z \in \Delta$ and all $f \in \mathcal{U}_{\alpha}^{H}$, we have

$$
\left|g^{\prime}(z)\right| \leq \frac{(1+r)^{\alpha-1}}{(1-r)^{\alpha+1}}
$$

for all $f=h+\bar{g} \in \mathcal{U}_{\alpha}^{H}$ and $z \in \Delta,|z|=r$. Consequently, $\mathcal{U}_{\alpha, c}^{H} \subset$ $\subset \mathcal{U}_{\alpha}^{H}$ is uniformly bounded on compact subsets of $\Delta$. According to the compactness principle, there exists a subsequence of $f_{n}$ (let us save the notation) which converges locally uniformly in $\Delta$ to a harmonic function $f_{0}$. Let us show that $f_{0} \in \mathcal{U}_{\alpha, c}^{H}$.

For $f \in \mathcal{U}_{\alpha}^{H}$ the following inequality holds (see [10])

$$
\frac{(1-r)^{2 \alpha-2}}{(1+r)^{2 \alpha+2}} \leq \frac{J_{f}(z)}{J_{f}(0)} \leq \frac{(1+r)^{2 \alpha-2}}{(1-r)^{2 \alpha+2}}, \quad|z|=r .
$$

Therefore for $f_{n} \in \mathcal{U}_{\alpha, c}^{H}$ we have

$$
J_{f_{n}}(z) \geq \frac{(1-r)^{2 \alpha-2}}{(1+r)^{2 \alpha+2}}\left(1-c^{2}\right)>0 .
$$

This implies $J_{f_{0}}(z)>0$ for all $z \in \Delta$. This means that the harmonic in $\Delta$ function $f_{0}$ is sense-preserving.

Next, we prove that $\overline{\text { ord }} f_{0} \leq \alpha$. Suppose not. Then, we may let $\overline{\text { ord }} f_{0}=\beta>\alpha$. Then, by the definition of the strong order, there exist a conformal automorphism $\varphi(z)=\frac{z+a}{1+\bar{a} z}$ of $\Delta$ and $\theta \in \mathbb{R}$ such that for harmonic function

$$
e^{-i \theta}\left(f_{0}\right)_{a}\left(z e^{i \theta}\right)=\frac{f_{0}\left(\varphi\left(z e^{i \theta}\right)\right)-f_{0}(\varphi(0))}{h_{0}^{\prime}(\varphi(0)) \varphi^{\prime}(0) e^{i \theta}}=\sum_{k=1}^{\infty}\left(A_{k} z^{k}+A_{-k} \bar{z}^{k}\right),
$$

$\left(A_{1}=1, f_{0}=h_{0}+\overline{g_{0}}\right)$ the inequality

$$
\frac{\left|A_{2}-A_{-1} \overline{A_{-2}}\right|}{1-\left|A_{-1}\right|^{2}}>\alpha+\frac{\beta-\alpha}{2}
$$

is valid.

For the automorphism $\varphi$ and the number $\theta$ denote

$$
e^{-i \theta}\left(f_{n}\right)_{a}\left(z e^{i \theta}\right)=\sum_{k=1}^{\infty}\left(A_{k}^{(n)} z^{k}+A_{-k}^{(n)} \bar{z}^{k}\right), \quad\left(A_{1}^{(n)}=1\right) .
$$


From locally uniform convergence of $f_{n}$ to $f_{0}$, the Weierstrass theorem on series of analytic functions, and inequality (4) it follows that for sufficiently large $n>N$

$$
\frac{\left|A_{2}^{(n)}-A_{-1}^{(n)} \overline{A_{-2}^{(n)}}\right|}{1-\left|A_{-1}^{(n)}\right|^{2}}>\alpha+\frac{\beta-\alpha}{2} .
$$

Hence if $n>N$ we have $\overline{\text { ord }} f_{n}>\alpha+\frac{\beta-\alpha}{2}$ and $f_{n} \notin \mathcal{U}_{\alpha, c}^{H}$. This contradiction proves the theorem.

In claim c) of Theorem B and claim e) of Theorem $\mathrm{C}$ some set of functions with the Hayman number $\delta^{0}=1$ (or $\delta_{0}=1$ for the theorem of decrease regularity) is described. These claims differ from the analytic case. In the analytic case $\delta^{0}=1$ and $\delta_{0}=1$ only for the functions $e^{i \theta} k_{\alpha}\left(z e^{-i \theta}\right)$, where $\theta \in \mathbb{R}, k_{\alpha}(z)$ is the function defined by [3] [7, [13, 14]. The following example shows that in the harmonic case this set has more complicated structure. We construct the example of functions $f$ of arbitrary strong order $\beta \geq 3 / 2$ with $\delta^{0}=1$. These functions are not equal to the function $q_{\theta}(z)$ from Theorem B. We use the Clunie and SheilSmall shear construction [15] (see also [16, ch. 3.4]) to give our example. Let us note that our construction is not stable. As one can show, if we multiply the coanalytic part $g$ of the function from our example by constant $k \in(0,1)$, then the strong order of the function changes stepwise and $\delta^{0} \neq 1$ for this function.

Example. Put $h^{\prime}(z)=\frac{(1+z)^{\alpha-1}}{(1-z)^{\alpha+2}}, g^{\prime}(z)=z h^{\prime}(z), z \in \Delta$. Let $\alpha \in[1, \infty)$ be fixed. If $\varphi(z)=\frac{z+a}{1+\bar{a} z}, a \in \Delta$, is an automorphism of $\Delta$, then for $f=h+\bar{g}$ we have

$$
f_{a}(z)=: F(z)=H(z)+\overline{G(z)}=\frac{h(\varphi(z))-h(\varphi(0))}{h^{\prime}(\varphi(0)) \varphi^{\prime}(0)}+\overline{\left(\frac{g(\varphi(z))-g(\varphi(0))}{h^{\prime}(\varphi(0)) \varphi^{\prime}(0)}\right)},
$$

where $H$ and $G$ are functions analytic in $\Delta$,

$$
\begin{gathered}
H^{\prime}(z)=\frac{h^{\prime}(\varphi(z)) \varphi^{\prime}(z)}{h^{\prime}(\varphi(0)) \varphi^{\prime}(0)} \text { and } \\
G^{\prime}(z)=\frac{g^{\prime}(\varphi(z)) \varphi^{\prime}(z)}{h^{\prime}(\varphi(0)) \varphi^{\prime}(0)}=\frac{\varphi(z) h^{\prime}(\varphi(z)) \varphi^{\prime}(z)}{h^{\prime}(\varphi(0)) \varphi^{\prime}(0)}
\end{gathered}
$$


Note that

$$
J_{F}(z)=\left|H^{\prime}(z)\right|^{2}-\left|G^{\prime}(z)\right|^{2}=\frac{\left|h^{\prime}(\varphi(z))\right|^{2}\left|\varphi^{\prime}(z)\right|^{2}\left(1-|\varphi(z)|^{2}\right)}{\left|h^{\prime}(\varphi(0))\right|^{2}\left|\varphi^{\prime}(0)\right|^{2}},
$$

and, in particular,

$$
J_{F}(0)=1-|\varphi(0)|^{2} .
$$

Therefore,

$$
\begin{gathered}
\frac{J_{F}(z)}{J_{F}(0)}=\frac{\left|1+\frac{z+a}{1+\bar{a} z}\right|^{2 \alpha-2}}{|1+a|^{2 \alpha-2}} \cdot\left|\frac{1-a}{1-\frac{z+a}{1+\bar{a} z}}\right|^{2 \alpha+4} \cdot \frac{\left(1-|a|^{2}\right)^{2}}{|1+\bar{a} z|^{4}} \times \\
\times\left(1-\left|\frac{z+a}{1+\bar{a} z}\right|^{2}\right) \frac{1}{\left(1-|a|^{2}\right)^{3}}= \\
=\frac{\left|1+z \frac{1+\bar{a}}{1+a}\right|^{2 \alpha-2}}{\left|1-z \frac{1-\bar{a}}{1-a}\right|^{2 \alpha+4}} \cdot \frac{|1+\bar{a} z|^{2}-|z+a|^{2}}{1-|a|^{2}}=\frac{\left|1+z \frac{1+\bar{a}}{1+a}\right|^{2 \alpha-2}}{\left|1-z \frac{1-\bar{a}}{1-a}\right|^{2 \alpha+4}}\left(1-|z|^{2}\right),
\end{gathered}
$$

by generalized Schwarz's lemma. Consequently, for $r \in(0,1)$

$$
\sup _{\substack{a \in \Delta \\|z|=r}} \frac{J_{F}(z)}{J_{F}(0)}=\frac{(1+r)^{2 \alpha-1}}{(1-r)^{2 \alpha+3}} \text {. }
$$

Therefore for $\beta=\alpha+\frac{1}{2}$, all $a \in \Delta$, and $|z|=r$ we get

$$
\frac{J_{F}(z)}{J_{F}(0)} \leq \frac{(1+r)^{2 \beta-2}}{(1-r)^{2 \beta+2}} .
$$

In [10] it was shown that for functions $f$ harmonic and sense-preserving in $\Delta$,

$$
\overline{\operatorname{ord}} f=\inf \left\{\beta: \frac{J_{F}(z)}{J_{F}(0)} \leq \frac{(1+|z|)^{2 \beta-2}}{(1-|z|)^{2 \beta+2}}, \quad \forall F=f_{a}, \forall z \in \Delta\right\} .
$$

From (5) and (6) we conclude that $\overline{\text { ord }} f \leq \beta=\alpha+\frac{1}{2}$. From Theorem $\mathrm{B}$ it follows that if for a function $f$ harmonic and sense-preserving in $\Delta$

$$
\lim _{r \rightarrow 1-}\left[\frac{J_{f}(z)}{J_{f}(0)} \frac{(1-r)^{2 \beta+2}}{(1+r)^{2 \beta-2}}\right]>0,
$$


then $\overline{\operatorname{ord}} f \geq \beta$. For the considered function $f$ the limit in $(7)$ equals 1 . Therefore, $\overline{\operatorname{ord}} f=\beta$ and

$$
\delta^{0}=\lim _{r \rightarrow 1-}\left[\frac{J_{f}(r)}{J_{f}(0)} \frac{(1-r)^{2 \beta+2}}{(1+r)^{2 \beta-2}}\right]=1 .
$$

It is interesting to find out if there exist functions with $\delta^{0}=1$ which are not equal to the function from the example and the functions $q_{\theta}(z)$.

Definition 4. A direction of intensive growth (d.i.g.) of a function $f(z)$ is a constant $\varphi \in[0 ; 2 \pi)$ such that

$$
\lim _{r \rightarrow 1-}\left[\frac{J_{f}\left(r e^{i \varphi}\right)}{J_{f}(0)} \frac{(1-r)^{2 \alpha+2}}{(1+r)^{2 \alpha-2}}\right]=\delta(f, \varphi)>0 .
$$

A direction of intensive decrease (d.i.d) of a function $f(z)$ is a constant $\varphi \in[0 ; 2 \pi)$ such that

$$
\lim _{r \rightarrow 1-}\left[\frac{J_{f}\left(r e^{i \varphi}\right)}{J_{f}(0)} \frac{(1+r)^{2 \alpha+2}}{(1-r)^{2 \alpha-2}}\right]=\delta^{\prime}(f, \varphi)<\infty .
$$

Since we study LIFs, it is important to know how d.i.g.-'s and d.i.d.-'s of a function $f(z)$ are changed under the transformation $e^{-i \theta} f_{a}\left(z e^{i \theta}\right)$. The case $a=0$ is trivial: a d.i.g. (d.i.d.) $\varphi-\theta$ of the function $e^{-i \theta} f\left(z e^{i \theta}\right)$ corresponds to the d.i.g. (d.i.d.) $\varphi$ of $f(z)$. In this situation $\delta(f(z), \varphi)=$ $=\delta\left(f\left(z e^{i \theta}\right), \varphi-\theta\right)$ (and $\left.\delta^{\prime}(f(z), \varphi)=\delta^{\prime}\left(f\left(z e^{i \theta}\right), \varphi-\theta\right)\right)$. It is also interesting to find out the relationship between the Hayman numbers of the functions $f$ and $f_{a}$ in general case. The following theorem concerns the non-obvious case $a \neq 0$.

Theorem 2. Let $f \in \mathcal{U}_{\alpha}^{H}$. Denote

$$
R(r)=\left|\frac{r e^{i \varphi}+a}{1+\bar{a} r e^{i \varphi}}\right|, \quad \gamma(r)=\arg \frac{r e^{i \varphi}+a}{1+\bar{a} r e^{i \varphi}}, \quad a \in \Delta, \quad r e^{i \varphi} \neq-a .
$$

1) $\varphi$ is a d.i.g. (d.i.d.) of the function $f_{a}(z)$ iff $\gamma$ is a d.i.g. (d.i.d.) of $f(z)$ and

$$
e^{i \varphi}=\frac{e^{i \gamma}-a}{1-\bar{a} e^{i \gamma}} ;
$$


2) for all $\gamma \in[0,2 \pi)$

$$
\lim _{r \rightarrow 1-}\left[\frac{J_{f}\left(r e^{i \gamma}\right)}{J_{f}(0)} \frac{(1-r)^{2 \alpha+2}}{(1+r)^{2 \alpha-2}}\right]=\lim _{r \rightarrow 1-}\left[\frac{J_{f}\left(R(r) e^{i \gamma(r)}\right)}{J_{f}(0)} \frac{(1-R(r))^{2 \alpha+2}}{(1+R(r))^{2 \alpha-2}}\right],
$$

and

$$
\lim _{r \rightarrow 1-}\left[\frac{J_{f}\left(r e^{i \gamma}\right)}{J_{f}(0)} \frac{(1+r)^{2 \alpha+2}}{(1-r)^{2 \alpha-2}}\right]=\lim _{r \rightarrow 1-}\left[\frac{J_{f}\left(R(r) e^{i \gamma(r)}\right)}{J_{f}(0)} \frac{(1+R(r))^{2 \alpha+2}}{(1-R(r))^{2 \alpha-2}}\right] .
$$

Here $\varphi$ and $\gamma$ are connected by (8).

3) if $\varphi$ is a d.i.g. of $f_{a}(z), \gamma$ is a d.i.g. of $f(z)$, and $\varphi$ is connected with $\gamma$ by (8), then

$$
\delta(f, \gamma)=\delta\left(f_{a}, \varphi\right) \frac{J_{f}(a)}{J_{f}(0)} \frac{\left(1-|a|^{2}\right)^{2 \alpha+2}}{\left|1+\bar{a} e^{i \varphi}\right|^{4 \alpha}}
$$

if $\varphi$ is a d.i.d. of $f_{a}(z), \gamma$ is a d.i.d. of $f(z)$, and $\varphi$ is connected with $\gamma$ by (8), then

$$
\delta^{\prime}(f, \gamma)=\delta^{\prime}\left(f_{a}, \varphi\right) \frac{J_{f}(a)}{J_{f}(0)} \frac{\left|1+\bar{a} e^{i \varphi}\right|^{4 \alpha}}{\left(1-|a|^{2}\right)^{2 \alpha-2}} .
$$

Proof. 1) Let $\varphi$ be a d.i.g. of $f_{a}(z)$. This means that there exists the limit

$$
\delta\left(f_{a}, \varphi\right)=\lim _{r \rightarrow 1-}\left[\frac{J_{f_{a}}\left(r e^{i \varphi}\right)}{J_{f_{a}}(0)} \frac{(1-r)^{2 \alpha+2}}{(1+r)^{2 \alpha-2}}\right]>0 .
$$

Note that

$$
J_{f_{a}}(z)=\frac{J_{f}\left(\frac{z+a}{1+\bar{a} z}\right)}{\left|h^{\prime}(a)\right|^{2}|1+\bar{a} z|^{4}}
$$

and

$$
J_{f_{a}}(0)=\frac{J_{f}(a)}{\left|h^{\prime}(a)\right|^{2}}
$$

Let us calculate the following limit, using (9) and (10),

$$
\begin{gathered}
\delta \stackrel{\text { def }}{=} \lim _{r \rightarrow 1-}\left[\frac{J_{f}\left(R(r) e^{i \gamma(r)}\right)}{J_{f}(0)} \frac{(1-R(r))^{2 \alpha+2}}{(1+R(r))^{2 \alpha-2}}\right]= \\
=\lim _{r \rightarrow 1-}\left[\frac{J_{f_{a}}\left(r e^{i \varphi}\right)}{J_{f}(0)}\left|h^{\prime}(a)\right|^{2}\left|1+\bar{a} r e^{i \varphi}\right|^{4} \frac{(1-r)^{2 \alpha+2}}{(1+r)^{2 \alpha-2}}\left(\frac{1-R(r)}{1-r}\right)^{2 \alpha+2}\right] .
\end{gathered}
$$


We have

$$
\lim _{r \rightarrow 1-} \frac{1-R(r)}{1-r}=\lim _{r \rightarrow 1-} R^{\prime}(r)=\frac{1-|a|^{2}}{\left|1+\bar{a} e^{i \varphi}\right|^{2}} .
$$

Using (11), we obtain

$$
\delta=\delta\left(f_{a}, \varphi\right) \frac{J_{f}(a)}{J_{f}(0)}\left|1+\bar{a} e^{i \varphi}\right|^{4}\left(\frac{1-|a|^{2}}{\left|1+\bar{a} e^{i \varphi}\right|^{2}}\right)^{2 \alpha+2}>0 .
$$

By (11), $\lim _{r \rightarrow 1-} R^{\prime}(r)>0$, therefore the function $R(r)$ increases on an interval $\left(r_{0}, 1\right)$. By Theorem B, for $r_{0}<r<r_{1}<1$

$$
\frac{J_{f}\left(R\left(r_{1}\right) e^{i \gamma\left(r_{1}\right)}\right)}{J_{f}(0)} \frac{\left(1-R\left(r_{1}\right)\right)^{2 \alpha+2}}{\left(1+R\left(r_{1}\right)\right)^{2 \alpha-2}} \leq \frac{J_{f}\left(R(r) e^{i \gamma\left(r_{1}\right)}\right)}{J_{f}(0)} \frac{(1-R(r))^{2 \alpha+2}}{(1+R(r))^{2 \alpha-2}} .
$$

Passing to the limit as $r_{1} \rightarrow 1-$ and using (8), we get

$$
\delta \leq \frac{J_{f}\left(R(r) e^{i \gamma}\right)}{J_{f}(0)} \frac{(1-R(r))^{2 \alpha+2}}{(1+R(r))^{2 \alpha-2}} .
$$

Thus,

$$
\delta(f, \gamma)=\lim _{r \rightarrow 1-}\left[\frac{J_{f}\left(R(r) e^{i \gamma}\right)}{J_{f}(0)} \frac{(1-R(r))^{2 \alpha+2}}{(1+R(r))^{2 \alpha-2}}\right] \geq \delta .
$$

Taking into account 12 , we conclude that $\gamma$ is a d.i.g. of $f(z)$.

Now let us consider the sets

$$
\begin{gathered}
A=\left\{e^{i \gamma}: \gamma \text { is a d.i.g. of } f(z)\right\}, \\
B=\left\{\frac{e^{i \varphi}+a}{1+\bar{a} e^{i \varphi}}: \varphi \text { is a d.i.g. of } f_{a}(z)\right\}, \\
C=\left\{e^{i \eta}: \eta \text { is a d.i.g. of }\left[f_{a}\right]_{(-a)}(z)\right\} .
\end{gathered}
$$

Here $\left[f_{a}\right]_{(-a)}(z)$ is the transformation (2) of the function $f_{a}$ with the parameter $-a$. If $\eta$ is a d.i.g. of $\left[f_{a}\right]_{(-a)}(z)$, then, as it was proved above,

$$
e^{i \eta}=\frac{e^{i \varphi}+a}{1+\bar{a} e^{i \varphi}}
$$

where $\varphi$ is a d.i.g. of $f_{a}(z)$. This implies that $C \subset B$. Let $\varphi$ be a d.i.g. of $f_{a}(z)$. Then

$$
e^{i \gamma}=\frac{e^{i \varphi}+a}{1+\bar{a} e^{i \varphi}}
$$


where $\gamma$ is a d.i.g. of $f(z)$. Thus $B \subset A$. Since $\left[f_{a}\right]_{(-a)}(z)=f(z)$, we have $A=C$ and, consequently, $A=B$. This completes the proof of the statement about d.i.g.-'s.

The statement about d.i.d.-'s is proved analogously.

2) Let us prove the first equality. If $\gamma$ is not a d.i.g. of $f(z)$, then

$$
\lim _{r \rightarrow 1-}\left[\frac{J_{f}\left(r e^{i \gamma}\right)}{J_{f}(0)} \frac{(1-r)^{2 \alpha+2}}{(1+r)^{2 \alpha-2}}\right]=0 .
$$

Thus, by (13),

$$
\delta \leq \lim _{r \rightarrow 1-}\left[\frac{J_{f}\left(R(r) e^{i \gamma}\right)}{J_{f}(0)} \frac{(1-R(r))^{2 \alpha+2}}{(1+R(r))^{2 \alpha-2}}\right]=0 .
$$

This implies $\delta=0$.

Now let us consider the case when $\gamma$ is a d.i.g. of $f(z)$. We have proved above that $\delta(f, \gamma) \geq \delta$ (see $(13)$ ). It remains to show that $\delta(f, \gamma) \leq \delta$.

Denote

$$
R_{1}(r)=\left|\frac{r e^{i \gamma}-a}{1-\bar{a} r e^{i \gamma}}\right|
$$

Since $\left[f_{a}\right]_{(-a)}(z)=f(z), \gamma$ is a d.i.g. of $\left[f_{a}\right]_{(-a)}(z)$, i. e.

$$
\delta\left(\left[f_{a}\right]_{(-a)}, \gamma\right)=\delta(f, \gamma)=\lim _{r \rightarrow 1-}\left[\frac{J_{\left[f_{a}\right]_{(-a)}}\left(r e^{i \gamma}\right)}{J_{f}(0)} \frac{(1-r)^{2 \alpha+2}}{(1+r)^{2 \alpha-2}}\right]>0 .
$$

Arguing as in the proof of claim 1), one can note that there exists

$$
\delta^{*} \stackrel{\text { def }}{=} \lim _{r \rightarrow 1-}\left[\frac{J_{f_{a}}\left(\frac{r e^{i \gamma}-a}{1-\bar{a} r e^{i \gamma}}\right)}{J_{f_{a}}(0)} \frac{\left(1-R_{1}(r)\right)^{2 \alpha+2}}{\left(1+R_{1}(r)\right)^{2 \alpha-2}}\right] .
$$

Apply 13 to the function $f_{a}(z)$, using $\left.(9), 10\right)$, and (11):

$$
\begin{gathered}
\delta^{*} \leq \lim _{r \rightarrow 1-}\left[\frac{J_{f_{a}}\left(r e^{i \varphi}\right)}{J_{f_{a}}(0)} \frac{(1-r)^{2 \alpha+2}}{(1+r)^{2 \alpha-2}}\right]= \\
=\lim _{r \rightarrow 1-}\left[\frac{J_{f}\left(\frac{r e^{i \varphi}+a}{1+\bar{a} r e^{i \varphi}}\right)}{J_{f}(a)\left|1+\bar{a} r e^{i \varphi}\right|^{4}} \frac{(1-R(r))^{2 \alpha+2}}{(1+R(r))^{2 \alpha-2}}\right] \cdot \lim _{r \rightarrow 1-}\left(\frac{1-r}{1-R(r)}\right)^{2 \alpha+2}=
\end{gathered}
$$




$$
=\frac{\delta J_{f}(0)}{J_{f}(a)\left|1+\bar{a} e^{i \varphi}\right|^{4}}\left(\frac{\left|1+\bar{a} e^{i \varphi}\right|^{2}}{1-|a|^{2}}\right)^{2 \alpha+2}=\frac{\delta J_{f}(0)}{J_{f}(a)} \frac{\left|1+\bar{a} e^{i \varphi}\right|^{4 \alpha}}{\left(1-|a|^{2}\right)^{2 \alpha+2}} .
$$

On the other hand, by (9),

$$
J_{f_{a}}\left(\frac{z-a}{1-\bar{a} z}\right)=\frac{J_{f}(z)}{\left|h^{\prime}(a)\right|^{2}\left|1+\bar{a} \frac{z-a}{1-\bar{a} z}\right|^{4}} .
$$

Thus, using (8), 10), and (11), we can write $\delta^{*}$ in the form

$$
\begin{aligned}
\delta^{*}=\lim _{r \rightarrow 1-} & {\left[\frac{J_{f}\left(r e^{i \gamma}\right)}{J_{f}(a)\left|1+\bar{a} \frac{r e^{i \gamma}-a}{1-\bar{a} r e^{i \gamma}}\right|^{4}} \frac{(1-r)^{2 \alpha+2}}{(1+r)^{2 \alpha-2}}\right] \times } \\
& \times \lim _{r \rightarrow 1-}\left(\frac{1-R_{1}(r)}{1-r}\right)^{2 \alpha+2}= \\
=\delta(f, \gamma) & \frac{J_{f}(0)}{J_{f}(a)\left|1+\bar{a} e^{i \varphi}\right|^{4}}\left(\frac{1-|a|^{2}}{\left|1-\bar{a} e^{i \gamma}\right|^{2}}\right)^{2 \alpha+2}= \\
= & \delta(f, \gamma) \frac{J_{f}(0)}{J_{f}(a)} \frac{\left|1+\bar{a} e^{i \varphi}\right|^{4 \alpha}}{\left(1-|a|^{2}\right)^{2 \alpha+2}} .
\end{aligned}
$$

Substituting

$$
\delta^{*}=\delta(f, \gamma) \frac{J_{f}(0)}{J_{f}(a)} \frac{\left|1+\bar{a} e^{i \varphi}\right|^{4 \alpha}}{\left(1-|a|^{2}\right)^{2 \alpha+2}}
$$

in (14), we get $\delta(f, \gamma) \leq \delta$. Therefore, $\delta(f, \gamma)=\delta$.

The second equality of claim 2 ) is proved analogously.

3) The formula, connected $\delta(f, \gamma)$ and $\delta\left(f_{a}, \varphi\right)$ is obtained from 112 , using $\delta=\delta(f, \gamma)$.

The second equality is proved analogously.

Theorem 2 implies the following

Remark. Let $f \in \mathcal{U}_{\alpha}^{H}$. For every $\varphi \in[0 ; 2 \pi)$ there exist $\delta(f, \varphi) \in[0 ; 1]$ and $\delta^{\prime}(f, \varphi) \in[1 ; \infty]$ such that for any circle or straight line $\Gamma \subset \Delta$, orthogonal to $\partial \Delta$ at the point $e^{i \varphi}$, we have

$$
\lim _{\Gamma \ni z \rightarrow e^{i \varphi}}\left[\frac{J_{f}(z)}{J_{f}(0)} \frac{(1-|z|)^{2 \alpha+2}}{(1+|z|)^{2 \alpha-2}}\right]=\delta(f, \varphi),
$$




$$
\lim _{\Gamma \ni z \rightarrow e^{i \varphi}}\left[\frac{J_{f}(z)}{J_{f}(0)} \frac{(1+|z|)^{2 \alpha+2}}{(1-|z|)^{2 \alpha-2}}\right]=\delta^{\prime}(f, \varphi),
$$

and the constants $\delta(f, \varphi), \delta^{\prime}(f, \varphi)$ do not depend on $\Gamma$.

By $\mathcal{U}_{\alpha}^{H}\left(\delta^{0}\right)$ denote the set of all functions from $\mathcal{U}_{\alpha}^{H}$ with the same Hayman number $\delta^{0}$ from Theorem B.

Let $\mathcal{U}_{\alpha}^{H}\left(\delta_{0}\right)$ be the set of all functions, having the Hayman number $\delta_{0}$ from Theorem $\mathrm{C}$.

Theorem 3. 1) If $f \in \mathcal{U}_{\alpha}^{H}\left(\delta^{0}\right), \delta^{0} \in(0 ; 1)$, then for every $\delta \in\left[\delta^{0}, 1\right)$ there exists $a \in \Delta$ such that $f_{a}(z) \in \mathcal{U}_{\alpha}^{H}(\delta)$.

2) If $f \in \mathcal{U}_{\alpha}^{H}\left(\delta_{0}\right), \delta_{0} \in(1 ; \infty)$, then for every $\delta^{\prime} \in\left(1, \delta^{0}\right]$ there exists $a \in \Delta$ such that $f_{a}(z) \in \mathcal{U}_{\alpha}^{H}\left(\delta^{\prime}\right)$.

Proof. By Theorem B, for any $\varphi \in[0 ; 2 \pi)$ there exists

$$
\lim _{r \rightarrow 1-}\left[\frac{J_{f}\left(r e^{i \varphi}\right)}{J_{f}(0)} \frac{(1-r)^{2 \alpha+2}}{(1+r)^{2 \alpha-2}}\right]=\delta(f, \varphi) .
$$

Let us fix $a \in \Delta \quad \varphi \in[0 ; 2 \pi)$. Denote $z=\frac{r e^{i \varphi}-a}{1-\bar{a} r e^{i \varphi}},|z|=R(r)$ and consider the limit

$$
\delta^{*}(\varphi) \stackrel{\text { def }}{=} \lim _{r \rightarrow 1-}\left[\frac{J_{f_{a}}(z)}{J_{f_{a}}(0)} \frac{(1-R(r))^{2 \alpha+2}}{(1+R(r))^{2 \alpha-2}}\right] .
$$

Let us calculate $\delta^{*}(\varphi)$, using $(9)$ and 10

$$
\begin{gathered}
\delta^{*}(\varphi)=\lim _{r \rightarrow 1-}\left[\frac{J_{f}\left(r e^{i \varphi}\right)}{J_{f}(a)\left|1+\bar{a} \frac{r e^{i \varphi}-a}{1-\bar{a} r e^{i \varphi}}\right|^{4}} \frac{(1-R(r))^{2 \alpha+2}}{(1+R(r))^{2 \alpha-2}}\right]= \\
=\lim _{r \rightarrow 1-}\left[\frac{J_{f}\left(r e^{i \varphi}\right)}{J_{f}(0)} \frac{(1-r)^{2 \alpha+2}}{(1+r)^{2 \alpha-2}} \frac{J_{f}(0)}{J_{f}(a)}\left(\frac{1-R(r)}{1-r}\right)^{2 \alpha+2}\right] \cdot \frac{1}{\left|1+\bar{a} \frac{r e^{i \varphi}-a}{1-\bar{a} r e^{i \varphi}}\right|} .
\end{gathered}
$$

By (11),

$$
\begin{aligned}
\delta^{*}(\varphi)= & \delta(f, \varphi) \frac{J_{f}(0)}{J_{f}(a)} \frac{\left(1-|a|^{2}\right)^{2 \alpha+2}}{\left|1-\bar{a} e^{i \varphi}\right|^{4 \alpha+4}} \frac{\left|1-\bar{a} e^{i \varphi}\right|^{4}}{\left(1-|a|^{2}\right)^{4}}= \\
& =\delta(f, \varphi) \frac{J_{f}(0)}{J_{f}(a)} \frac{\left(1-|a|^{2}\right)^{2 \alpha-2}}{\left|1-\bar{a} e^{i \varphi}\right|^{4 \alpha}} \leq
\end{aligned}
$$




$$
\leq \lim _{R(r) \rightarrow 1-}\left[\frac{M\left(R(r), J_{f_{a}}\right)}{J_{f_{a}}(0)} \frac{(1-R(r))^{2 \alpha+2}}{(1+R(r))^{2 \alpha-2}}\right] \stackrel{\text { def }}{=} \delta_{a} .
$$

Let $\varphi$ be equal to d.m.g. $\varphi^{0}$ of $f(z)$ and $a=\rho e^{i \varphi^{0}}$. Then $\delta(f, \varphi)=\delta^{0}$ and

$$
\delta^{0} \frac{J_{f}(0)}{J_{f}\left(\rho e^{i \varphi^{0}}\right)} \frac{\left(1-\rho^{2}\right)^{2 \alpha-2}}{(1-\rho)^{4 \alpha}}=\delta^{0} \frac{J_{f}(0)}{J_{f}\left(\rho e^{i \varphi^{0}}\right)} \frac{(1+\rho)^{2 \alpha-2}}{(1-\rho)^{2 \alpha-2}} \leq \delta_{a} .
$$

By Theorem B, there exists a d.m.g. $\varphi_{1} \in[0 ; 2 \pi)$ of $f_{a}(z)$ such that

$$
\begin{gathered}
\delta_{a}=\lim _{r \rightarrow 1-}\left[\frac{J_{f_{a}}\left(r e^{i \varphi^{0}}\right)}{J_{f_{a}}(0)} \frac{(1-r)^{2 \alpha+2}}{(1+r)^{2 \alpha-2}}\right]= \\
=\lim _{r \rightarrow 1-}\left[\frac{J_{f}\left(\frac{r e^{i \varphi_{1}}+a}{1+\bar{a} r e^{i \varphi_{1}}}\right)}{J_{f}(a)\left|1+\bar{a} r e^{i \varphi_{1}}\right|^{4}} \frac{(1-r)^{2 \alpha+2}}{(1+r)^{2 \alpha-2}}\right] .
\end{gathered}
$$

Denote $R_{1}(r) e^{i \gamma_{1}(r)}=\frac{r e^{i \varphi_{1}}+a}{1+\bar{a} r e^{i \varphi_{1}}}$, where $\gamma_{1}(r)$ is a real-valued function. Then, using (11) for $R(r)=R_{1}(r)$, we obtain

$$
\begin{gathered}
\delta_{a} \leq \lim _{r \rightarrow 1-}\left[\frac{M\left(R_{1}(r), J_{f}\right)}{J_{f}(a) \mid 1+\bar{a} r e^{\left.i \varphi_{1}\right|^{4}}} \frac{(1-r)^{2 \alpha+2}}{(1+r)^{2 \alpha-2}}\right]= \\
=\lim _{r \rightarrow 1-}\left[\frac{M\left(R_{1}(r), J_{f}\right)}{J_{f}(0)} \frac{\left(1-R_{1}(r)\right)^{2 \alpha+2}}{\left(1+R_{1}(r)\right)^{2 \alpha-2}}\right] \times \\
\times \frac{J_{f}(0)}{J_{f}(a)} \frac{1}{\left|1+\bar{a} e^{i \varphi_{1}}\right|^{4}} \cdot \lim _{r \rightarrow 1-}\left(\frac{1-r}{1-R_{1}(r)}\right)^{2 \alpha+2}= \\
=\delta^{0} \frac{J_{f}(0)}{J_{f}(a)} \frac{1}{\left|1+\bar{a} e^{i \varphi_{1}}\right|^{4}}\left(\frac{\left|1+\bar{a} e^{i \varphi_{1}}\right|^{2}}{1-|a|^{2}}\right)^{2 \alpha+2}=\delta^{0} \frac{J_{f}(0)}{J_{f}(a)} \frac{\left|1+\bar{a} e^{i \varphi_{1}}\right|^{4 \alpha}}{\left(1-|a|^{2}\right)^{2 \alpha+2}} \leq \\
\leq \delta^{0} \frac{J_{f}(0)}{J_{f}(a)} \frac{(1+\rho)^{4 \alpha}}{\left(1-\rho^{2}\right)^{2 \alpha+2}}=\delta^{0} \frac{J_{f}(0)}{J_{f}(a)} \frac{(1+\rho)^{2 \alpha-2}}{(1-\rho)^{2 \alpha+2}} .
\end{gathered}
$$

Taking into account inequality (15), we get

$$
\delta^{0} \frac{J_{f}(0)}{J_{f}\left(\rho e^{i \varphi^{0}}\right)} \frac{(1+\rho)^{2 \alpha-2}}{(1-\rho)^{2 \alpha+2}}=\delta_{a} .
$$


Since the continuous function $\frac{J_{f}(0)}{J_{f}\left(\rho e^{i \varphi^{0}}\right)} \frac{(1+\rho)^{2 \alpha-2}}{(1-\rho)^{2 \alpha+2}}$ decreases on $\rho$, equals 1 as $\rho=0$, and tends to zero as $\rho \rightarrow 1-$, then we can find $\rho \in[0 ; 1)$ such that $\delta_{a}$ takes preassigned value from $\left[\delta^{0} ; 1\right)$.

Claim 2 of the theorem is proved analogously.

In [7] (see also [17, [14]) it was proved that the set of all d.i.g.-'s and d.i.d.-'s of a given analytic function is at most countable. The following theorem shows that this statement is true for set of d.i.g.-'s of harmonic function too. But we don't know whether this fact is true for set of d.i.d.-'s.

Theorem 4. Let $f \in \mathcal{U}_{\alpha}^{H}$. Then the set of all d.i.g.-'s of $f$ is at most countable.

Proof. If $f=h+\bar{g} \in \mathcal{U}_{\alpha}^{H}$, then $\overline{\text { ord }} h \leq \alpha$. Since

$$
J_{f}(z)=\left|h^{\prime}(z)\right|^{2}-\left|g^{\prime}(z)\right|^{2} \leq\left|h^{\prime}(z)\right|^{2}
$$

for all $z \in \Delta$, then for $\varphi \in[0,2 \pi)$ and $r \in[0,1)$

$$
\frac{J_{f}\left(r e^{i \varphi}\right)}{J_{f}(0)} \frac{(1-r)^{2 \alpha+2}}{(1+r)^{2 \alpha-2}} \leq\left[\left|h^{\prime}\left(r e^{i \varphi}\right)\right| \frac{(1-r)^{\alpha+1}}{(1+r)^{\alpha-1}}\right]^{2} \frac{1}{J_{f}(0)}
$$

By Theorem B and theorem of growth regularity from [7], there exist the limits

$$
\delta(f, \varphi)=\lim _{r \rightarrow 1-}\left[\frac{J_{f}\left(r e^{i \varphi}\right)}{J_{f}(0)} \frac{(1-r)^{2 \alpha+2}}{(1+r)^{2 \alpha-2}}\right],
$$

and

$$
\tilde{\delta}(h, \varphi)=\lim _{r \rightarrow 1-}\left[\left|h^{\prime}\left(r e^{i \varphi}\right)\right| \frac{(1-r)^{2 \alpha+2}}{(1+r)^{2 \alpha-2}}\right] .
$$

From 16 we get $\delta(f, \varphi) \leq \frac{\tilde{\delta}^{2}(h, \varphi)}{J_{f}(0)}$. If $\varphi$ is a d.i.g. of $f$, then $\delta(f, \varphi)>0$. Consequently, $\tilde{\delta}(h, \varphi)>0$ and $\varphi$ is a d.i.g. of $h$. Therefore the set $V$ of all d.i.g.-'s of $f$ is contained in the set $W$ of all d.i.g.-'s of $h$. As it was proved in [7], $W$ is at most countable. Hence $V$ is at most countable too.

Acknowledgment. This work was supported by RFBR (projects N 1401-00510f, N 14-01-92692). The authors thank S. Yu. Graf and S. Ponnusamy for valuable comments on improving the paper. 


\section{References}

[1] Hayman W. K. Some applications of the transfinite diameter to the theory of functions. J. Anal. Math., 1951, no. 1, pp. 155-179.

[2] Krzyż J. On the maximum modulus of univalent function. Bull. Pol. Acad. Sci. Math., 1955, vol. CI, no. 3, pp. 203-206.

[3] Bieberbach L. Einführung in die konforme Abbildung. Sammlung Göschen, Band 768/786a, 1967.

[4] Hayman W. K. Multivalent functions. Cambridge University Press, 1994.

[5] Pommerenke Ch. Linear-invariante Familien analytischer Funktionen. I. Math. Ann., 1964, vol. 155, pp. 108-154. DOI: 10.1007/BF01344077.

[6] Campbell D. M. Applications and proofs of a uniqueness theorems for linear invariant families of finite order. Rocky Mountain J. Math., 1974, vol. 4, no. 4, pp. 621-634. DOI: 10.1216/RMJ-1974-4-4-621.

[7] Starkov V. V. Regularity theorems for universal linearly invariant families of functions. Serdica Math. J., 1985, vol. 11, no. 3, pp. 299-318. (in Russian).

[8] Schaubroeck L. E. Subordination of planar harmonic functions. Complex Variables, 2000, vol. 41, is. 2, pp. 163-178. DOI: $10.1080 / 17476930008815245$

[9] Sheil-Small T. Constants for planar harmonic mappings. J. Lond. Math. Soc. (2), 1990, vol. 42, pp. 237-248. DOI: 10.1112\%2Fjlms\%2Fs2-42.2.237.

[10] Sobczak-Kneć M., Starkov V. V., Szynal J. Old and new order of linear invariant family of harmonic mappings and the bound for Jacobian. Ann. Univ. Mariae Curie-Sklodowska Sect. A, 2012, vol. LXV, no. 2, pp. 191202. DOI: $10.2478 / \mathrm{v} 10062-011-0024-3$

[11] Ganenkova E. G., Starkov V. V. Regularity theorems for harmonic functions. J. Appl. Anal., 2014, vol. 21, is. 1, pp. 25-36. DOI: 10.1515/jaa-20150003.

[12] Graf S. Yu. Regularity theorems for Jacobian in linearly and affine invariant families of harmonic mappings. Application of the functional analysis in the approximation theory, 2014, pp. 10-21. (in Russian).

[13] Ganenkova E. G. A theorem of decrease regularity in linearly invariant families of functions. Tr. Petrozavodsk. Gos. Univ. Ser. Mat, 2006, vol. 13, pp. 46-59 (in Russian).

[14] Ganenkova E. G. A theorem on the regularity of decrease in linearly invariant families of functions. Russian Mathematics (Izvestiya VUZ. Matematika), 2007, vol. 51, no. 2, pp. 71-74. DOI: 10.3103/S1066369X07020090. 
[15] Clunie J., Sheil-Small T. Harmonic univalent functions. Ann. Acad. Sci. Fenn. Math., 1984, vol. 9, pp. 3-25. DOI: 10.5186/aasfm.1984.0905\#sthash.GEE2gFK0.dpuf.

[16] Duren P. Harmonic mappings in the plane. Cambridge university press, 2004.

[17] Godula J., Starkov V. V. Linear invariant families. Tr. Petrozavodsk. Gos. Univ. Ser. Mat., 1998, vol. 5, p. 3-96 (in Russian).

Received May 14, 2015.

In revised form, September 3, 2015.

Petrozavodsk State University

33, Lenina st., 185910 Petrozavodsk, Russia

E-mail: g_ek@inbox.ru, Vstar@psu.karelia.ru 